\title{
Insights into dog owner perspectives on risks, benefits, and nutritional value of raw diets compared to commercial cooked diets
}

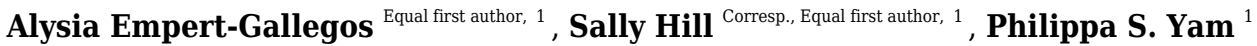 \\ ${ }^{1}$ School of Veterinary Medicine, University of Glasgow, Glasgow, United Kingdom \\ Corresponding Author: Sally Hill \\ Email address: 2217479p@student.gla.ac.uk
}

Background. The practice of feeding a raw meat-based diet (RMBD) to dogs is a topic of increasing interest to owners and veterinary professionals alike. Despite the research around the practice, particularly about the risk of nutritional imbalances and microbial contamination, an increasing number of dog owners are adopting a raw diet for their dogs. This study expands the research into owner motivations for feeding RMBDs and cooked diets and asks them their opinions about risk and nutritional value.

Methods. An anonymized, online, internationally accessible questionnaire was developed to ascertain owner perspectives on the risks, benefits, and nutritional value of commercially prepared and homemade RMBDs as compared with commercially prepared cooked diets (CCDs).

Results. The questionnaire was completed by 419 dog owners of diverse backgrounds across the world. Of the participants, $25.3 \%$ fed RMBDs. Just over $70.0 \%$ of all participants had spoken to their veterinarian about their dog's nutrition. Owners who fed RMBDs ranked their veterinarian's knowledge lower and their own knowledge of canine nutrition higher than owners who fed CCDs. They rated commercial and homemade RMBDs as highly nutritious $83.5 \%$ and $73.6 \%$ of the time, respectively, while only $12.5 \%$ rated CCDs as highly nutritious. Owners who fed CCDs ranked RMBDs as highly nutritious less often, but also only ranked CCDs as highly nutritious $52.7 \%$ of the time. All participants agreed that CCDs were low risk to human health. Owners who fed RMBDs ranked raw diets as highly risky to human or dog health under $20.0 \%$ of the time but deemed CCDs risky to animal health over $65.0 \%$ of the time. When asked about benefits of raw diets, the most repeated words offered by owners were "health", "better", "coat" and "teeth". The most repeated risks presented were "bacteria", "nutrition", "risk" and "Salmonella". Owners who fed RMBDs tended to use vague terminology like "health" and "better" when asked why they fed a raw diet. Owners who did not feed RMBDs used more specific terminology like "expensive", "time" and "risk" when asked why they did not feed a raw diet. Overall, the two groups differed in their perceptions around RMBD and CCD feeding, which highlights the need for a better line of communication and education between veterinarians and owners. 
1 Insights into dog owner perspectives on risks, 2 benefits, and nutritional value of raw diets compared 3 to cooked diets

\footnotetext{
4 Alysia Empert-Gallegos*1, Sally Hill*1 and Philippa S. Yam ${ }^{1}$

5 'University of Glasgow School of Veterinary Medicine, Glasgow, UK

$6 *$ These authors contributed equally to the work

7

8 Corresponding Author:

9 Sally Hill ${ }^{1}$

10 Email address: 2217479p@student.gla.ac.uk
} 
11

12

13

14

15

16

17

18

19

20

21

22

23

24

25

26

27

28

29

30

31

32

33

34

35

36

37

38

39

40

41

42

43

44

45

46

47

48

49

50

51

52

53

\section{Abstract}

Background. The practice of feeding a raw meat-based diet (RMBD) to dogs is a topic of increasing interest to owners and veterinary professionals alike. Despite the research around the practice, particularly about the risk of nutritional imbalances and microbial contamination, an increasing number of dog owners are adopting a raw diet for their dogs. This study expands the research into owner motivations for feeding RMBDs and cooked diets and asks them their opinions about risk and nutritional value.

Methods. An anonymized, online, internationally accessible questionnaire was developed to ascertain owner perspectives on the risks, benefits, and nutritional value of commercially prepared and homemade RMBDs as compared with commercially prepared cooked diets (CCDs).

Results. The questionnaire was completed by 419 dog owners of diverse backgrounds across the world. Of the participants, $25.3 \%$ fed RMBDs. Just over $70.0 \%$ of all participants had spoken to their veterinarian about their dog's nutrition. Owners who fed RMBDs ranked their veterinarian's knowledge lower and their own knowledge of canine nutrition higher than owners who fed CCDs. They rated commercial and homemade RMBDs as highly nutritious $83.5 \%$ and $73.6 \%$ of the time, respectively, while only $12.5 \%$ rated CCDs as highly nutritious. Owners who fed CCDs ranked RMBDs as highly nutritious less often, but also only ranked CCDs as highly nutritious $52.7 \%$ of the time. All participants agreed that CCDs were low risk to human health. Owners who fed RMBDs ranked raw diets as highly risky to human or dog health under $20.0 \%$ of the time but deemed CCDs risky to animal health over $65.0 \%$ of the time.

When asked about benefits of raw diets, the most repeated words offered by owners were "health", "better", "coat" and "teeth". The most repeated risks presented were "bacteria", "nutrition", "risk" and "Salmonella". Owners who fed RMBDs tended to use vague terminology like "health" and "better" when asked why they fed a raw diet. Owners who did not feed RMBDs used more specific terminology like "expensive", "time" and "risk" when asked why they did not feed a raw diet. Overall, the two groups differed in their perceptions around RMBD and CCD feeding, which highlights the need for a better line of communication and education between veterinarians and owners.

\section{Introduction}

The practice of feeding a raw meat-based diet (RMBD) to dogs is a topic of increasing interest to owners and veterinary professionals alike, with Google searches for 'raw dog food' quadrupling over the last ten years. ${ }^{1}$ Estimates of the percentage of dog owners who feed RMBDs vary, but experts agree that the practice is increasing in both the United States and Europe. ${ }^{2}$

As the feeding of RMBDs has become more common there has been an increase in research on the safety of the practice, particularly the potential for nutritional imbalances and the microbiological risks associated with raw meat. Leading veterinary organizations, from the American Veterinary Medical Association to the World Small Animal Veterinary Association, discourage the feeding of RMBDs, but given its increasing popularity, some owners are clearly not heeding their warnings. ${ }^{3,4}$ Despite this increased popularity and interest in RMBDs, scant 
54 research has been published on dog owner opinions regarding this type of feeding and comparing

55 it to the feeding of commercially prepared cooked diets (CCDs).

56

57 This study was undertaken with the aim to better understand dog owner perspectives on risks,

58 benefits, and nutritional value of raw diets compared to cooked diets. We hypothesized that

59 owners who feed primarily raw diets to their dogs will perceive the practice to pose less risk to

60 both human and animal health than those who feed a non-raw diet. We also anticipated that those

61 who feed raw diets will perceive them as more or equally nutritious as CCDs.

62

\section{Materials \& Methods}

64 Approval to conduct the project was sought and granted by the University of Glasgow College of

65 Medical, Veterinary \& Life Sciences Ethics Committee for Non-Clinical Research Involving

66 Human Participants (Approval Ref: 200180125).

67

68

An anonymized, online, internationally accessible, open questionnaire was developed using Google Forms. The questionnaire was tested against peers to assess usability, technical functionality, comprehension, cohesiveness, flow, and length before fielding the study. The questionnaire consisted of 11 sections. The first section included a description of the questionnaire including time estimate for completion, stated the terms of consent, and included contact information for the researchers. Any person over the age of 18 who was the primary owner or caregiver of a pet dog was invited to participate as the target population. Participation was completely voluntary and required participants to consent to continue with the questionnaire. Participants were not required to sign in or belong to any network in order to participate. No incentives for participation were offered. The second section collected demographics about the dog owner. Sections 3-10 included dichotomous, categorical, ordinal and free-text box questions. Sections 3-10 included 1-4 questions each, with an average of 3 questions per section. Section 11 consisted of a brief appreciation for their time and ended the questionnaire. Participants were allowed to skip any question or answer free-text questions with "NA" (not applicable) and they were able to navigate forwards and backwards before submitting the questionnaire. Questions asked about general feeding trends and opinions, rather than asking for owners to respond about

Results were automatically collected by the Google Forms software and were exported from the internet when the data collection was finished.

After obtaining permission from page administrators, a live, sharable link to the questionnaire was initially posted four times on dog-centric community pages on Facebook; Dogspotting (1.7 million members) and Dogspotting Society (974,000 members). Facebook users frequent these types of pages to view and share photos or videos of dogs and information about dog ownership. 
94 researchers that the survey was posted elsewhere on Facebook by individuals other than the

95 researchers. The link remained live for 14 weeks during the summer of 2019.

96

97

98

Discrete data were compiled and analyzed using Microsoft Excel. Statistical significance was determined using chi-squared testing with a p-value of less than 0.001 unless specifically noted. When presenting percentages to compare cooked and raw feeding groups, summation of

100

101

102

103

104

105

106

107

108

109

110

111

112

113

114

115

116

117

118

119

120

121

122

123

124

125

126

127

128

129

130

131

132 percentages is always $100 \%$. Qualitative analysis of the fill-in data was undertaken using the RStudio text mining (tm) package and word cloud generator package (wordcloud) to discover relevant word frequencies and visually represent the data. Qualitative data were mined to exclude common English stop words and combine words with the same stem (e.g. risk and risky) as well as words with an identical meaning (e.g. stool, feces, faeces, poop, poo).

\section{Results}

\section{Demographics}

Four hundred and nineteen people of variable age responded to the survey. Most respondents were female ( $\mathrm{n}=393,93.6 \%$ ), omnivorous $(\mathrm{n}=357,85.6 \%$ ), and did not work as part of the animal industry or animal related field $(\mathrm{n}=306,73.2 \%)$. Only a minority of respondents lived in households with immunocompromised individuals ( $\mathrm{n}=33,7.9 \%)$, pregnant women $(\mathrm{n}=10,2.4 \%)$ or children under ten years of age $(n=43,10.3 \%)$. Our survey participants hailed from sixteen countries on five continents, with the United States ( $\mathrm{n}=206,58.4 \%)$ and the United Kingdom $(\mathrm{n}=97,27.5 \%)$ as the most represented and second most represented countries, respectively. (Table 1).

\section{Establishment of Diet}

When asked how owners established their dog's diet, 24.4\% ( $\mathrm{n}=101)$ of respondents said they followed a recommendation from a veterinarian, veterinary nurse or veterinary technician. Information published online from a non-veterinary source $(\mathrm{n}=54,13.0 \%)$ and information published by a veterinarian or veterinary nutritionist $(\mathrm{n}=50,12.1 \%)$ were also popular choices. The remainder of respondents $(\mathrm{n}=209,50.5 \%)$ established their dog's diet in myriad other ways, like recommendations from friends and family, tradition (what they have always fed), recommendation of the breeder or rescue/shelter and recommendation from breed specific literature, among others (101 discrete answers were offered).

Owners were asked what their dogs ate most of the time (their main diet). The majority of respondents fed their dog a CCD as the main diet, in the forms of dry/kibble or wet/canned/sachet $(n=267,63.7 \%)$. Commercially prepared raw food $(n=54,12.9 \%)$ was the next most popular choice, followed by a homemade raw diet $(\mathrm{n}=52,12.4 \%)$, a prescription diet for a medical condition $(n=30,7.2 \%)$ and a homemade cooked diet $(n=16,3.8 \%)$. For the purposes of this paper, owners who fed predominantly raw diets (as their main diet) in any form 
133 will herein be referred to as "raw feeders" while owners who fed predominantly cooked diets in

134

135

136

137

138

139

140

141

142

143

144

145

146

147

148

149

150

151

152

153

154

155

156

157

158

159

160

161

162

163

164

165

166

167

168

169

170

171

172

any form will be referred to as "cooked feeders".

\section{Perceptions of Diets}

Respondents were asked to rate various diets on scales of 1 to 5 (where 1 is lowest and 5 is highest) in terms of perceived nutritional value, as well as risks to both human and dog health; the diets they rated were CCDs, commercially prepared RMBDs, and homemade RMBDs.

When asked about nutrition, raw feeders were more likely to rate RMBDs as highly nutritious (4 or 5 out of 5) than cooked feeders were ( $<<0.001$ for all comparisons made), and made only a small distinction between commercial or homemade RMBDs, with $83.5 \%(\mathrm{n}=79)$ of raw feeders rating commercial RMBDs as highly nutritious and $73.6 \%(n=78)$ rating homemade RMBDs as highly nutritious. In contrast, cooked feeders made a greater distinction between commercial and homemade diets than between cooked and raw; $52.7 \%(\mathrm{n}=164)$ of cooked feeders rated CCDs as highly nutritious and $51.6 \%(\mathrm{n}=119)$ gave the same rating to commercial RMBDs. Only $35.7 \%$ $(\mathrm{n}=106)$ of cooked feeders rated homemade RMBDs as highly nutritious, and only $12.5 \%(\mathrm{n}=13)$ of raw feeders rated CCDs as highly nutritious (Figure 1).

When asked about risk, all participants rated CCDs as low risk to humans; there was no statistical difference between the groups $(\mathrm{p}=0.93)$. However, cooked feeders perceived RMBDs as riskier to both their dog's health and human health than raw feeders did ( $p<0.001$ for all). Both groups viewed CCDs as riskier to dog health than to human health: while approximately $15.0 \%$ of both groups thought there was a high risk to human health (cooked feeders $n=17$, raw feeders $n=7$ ), there was a significant difference in perception of risk to dog health between groups. Forty-five cooked feeders $(25.1 \%)$ rated CCDs as highly risky to dog health. The results from raw feeders were more extreme: $65.3 \%(n=54)$ of them rated CCDs as highly risky to dog health. (Figure 1).

\section{Perceptions of Nutritional Knowledge}

Two hundred and ninety-seven (70.9\%) respondents had discussed nutrition with their veterinarian. When asked to rank how knowledgeable they felt their veterinarian was about nutrition where 1 was least knowledgeable and 5 was most knowledgeable, 59.9\% $(n=249)$ of respondents gave their veterinarian a 4 or 5 out of 5 . When asked to rank how knowledgeable owners felt they were about their dog's nutrition, $61.9 \%(n=260)$ gave themselves 4 or 5 out of 5 . Further analysis of this data shows a significant difference in responses given by raw feeders and cooked feeders. When asked to rank their own nutritional knowledge on a scale of 1 to $5,65.2 \%$ $(\mathrm{n}=174)$ of cooked feeders ranked themselves as 4 or 5 out of 5 , while $86.0 \%(\mathrm{n}=85)$ of raw feeders gave themselves the same score $(\mathrm{p}<0.001)$. Only $45.5 \%(\mathrm{n}=33)$ of raw feeders gave their veterinarian a 4 or 5 on the scale, whereas $78.0 \%(n=215)$ of cooked feeders ranked their veterinarian as 4 or 5 out of $5(\mathrm{p}<0.001)$ (Figure 1$)$. 


\section{Free Text Answers}

176

Owners provided a variety of opinions via free-text boxes when asked about perceived benefits

177

178

179

180

181

182

183

184

185

186

187

188

189

190

191

192

193

194

195

196

197

198

199

200

201

202

203

204

205

206

207

208

209

210

211

212

and risks of raw feeding as well as why they chose to feed a RMBD or why they chose not to feed a RMBD to their dog. These perceptions are visually summarized in the word clouds (Figures 2-5). The most repeated words for benefits of RMBDs were "health" (frequency (f)= 105), "better" ( $\mathrm{f}=104)$, "coat" $(\mathrm{f}=59)$, and "teeth" ( $\mathrm{f}=50)$ (Figure 2) and the most repeated words for risks were "bacteria" ( $f=91)$, "nutrition" $(f=72)$, "risk" $(f=63)$, and "Salmonella" $(f=39)$ (Figure 3). When asked why they chose to feed raw, owners most often used the words "health" $(\mathrm{f}=54)$, "better" ( $\mathrm{f}=23)$, "nutrition" ( $\mathrm{f}=20)$ " and "coat" $(\mathrm{f}=14)$ (Figure 4). Conversely, when asked why they chose not to feed raw, the most common words were "expensive" (f=59), "time" $(\mathrm{f}=45)$, "risk" ( $\mathrm{f}=42)$, and "convenience" ( $\mathrm{f}=36)$ (Figure 5).

\section{Discussion}

Our demographic data indicates that our survey respondents were overwhelmingly female $(n=393,93.6 \%)$ and seems to be a typical result in surveys of pet owners. ${ }^{5}$ Though we did have responses from a breadth of countries, the United States and the United Kingdom were the two most represented countries, which is an expected result given that our questionnaire was only available in English.

Comparing raw feeders with cooked feeders revealed many key distinctions in opinion and perception between the two groups. To start, raw feeders and cooked feeders differed in their assessments of the nutritional quality of various diets. As we hypothesized, raw feeders perceived homemade and commercial RMBDs both as highly nutritious, making little distinction between the two preparations. This perception is not supported by scientific evidence; numerous studies have demonstrated the risks of nutritional imbalances inherent in homemade diets. ${ }^{6-9}$ Even homemade diets formulated by veterinarians have been shown to sometimes be incomplete though they tend to have fewer and less severe deficiencies than those formulated by nonveterinarians. ${ }^{6}$ Homemade diets should always be formulated in consultation with boardcertified veterinary nutritionists to ensure they are properly balanced. A wide array of medical conditions can be caused by improper nutrient balance including nutritional secondary hyperparathyroidism, developmental orthopedic conditions, and even canine nutritional hyperthyroidism; there are multiple documented cases of dogs developing these conditions as a result of eating improperly balanced homemade RMBDs. ${ }^{10-13}$ These risks are concerning, especially as a recent international study of pet owners found that $89 \%$ of raw-feeding dog owners fed homemade raw diets. ${ }^{14}$ A separate survey aimed specifically at raw feeders found that only $15 \%$ of respondents formulated their dog's RMBD with guidance from a veterinarian or nutritionist. ${ }^{15}$ The fact that raw feeders are not making a distinction between the nutritional quality of homemade and commercial RMBDs and that many seem to be formulating their dog's diet without appropriate guidance suggests a need for further owner education on the risks of

PeerJ reviewing PDF | (2020:06:49841:2:0:NEW 21 Oct 2020) 
213 feeding an improperly balanced homemade diet. By comparison, the majority of cooked feeders

214 did distinguish between their assessments of the two preparations, with few of them rating

215 homemade RMBDs as highly nutritious and a larger number rating both commercial RMBDs

216 and CCDs as highly nutritious. It is worth noting that a RMBD being commercially produced is

217 not a guarantee that it is nutritionally balanced, particularly given that legal standards for pet

218 food vary from country to country. The WSAVA recommends only feeding commercial pet food

219 (raw or cooked) from companies that meet specific standards, including the full-time

220 employment of a board-certified veterinary nutritionist. ${ }^{16}$

221

222 Unsurprisingly only one in eight raw feeders viewed CCDs as highly nutritious. Cooked feeders

223 demonstrated interesting perceptions of CCDs, with just over half of them $(n=164,52.7 \%)$ rating

224 CCDs as highly nutritious; that is an alarmingly low number considering this is the diet they

225 chose to feed to their dogs. When we combine these findings with the top reasons that owners

226 provided for not feeding RMBDs ("time", "expensive", "convenience"), we can postulate that

227 diet choice is multifactorial and that lifestyle factors may be playing a larger role than nutritional

228 value for some owners. This is an area that begs further research to understand exactly why

229 cooked feeders are choosing to feed a diet they do not view as particularly high in nutritional

230 value.

231

232 When it comes to risks associated with raw and cooked diets, raw feeders and cooked feeders

233 again had different perceptions. Cooked feeders were far more likely to rate both commercial

234 and homemade RMBDs as higher risk to both dog and human health than CCDs. This is

235 reassuring as it demonstrates an awareness of the published dangers surrounding the handling

236 and consumption of raw meat products. One of those risks is of zoonotic infection with

237 Salmonella, which is one of the most commonly found pathogens in RMBDs and may pose a

238 greater threat to owners than their pets; dogs have been found less likely to exhibit clinical signs

239 of Salmonella infections even while shedding the bacteria into their environments, and multiple

240 studies have demonstrated higher incidence of Salmonella shedding in dogs fed RMBDs. ${ }^{17-20}$

241 This risk is not necessarily mitigated by strict hygiene standards; Salmonella species have been

242 shown to persist in dog bowls used for RMBDs, even after being cleaned in a dishwasher at

$24385^{\circ} \mathrm{C}$ or scrubbed and soaked in bleach. ${ }^{21}$ Salmonella is the most common pathogenic risk from

244 raw feeding cited by our respondents, though it is by no means the only risk associated with

245 RMBDs; other examples of pathogens found in studies of RMBDs include the bacteria

246 Escherichia coli species, Campylobacter species, Clostridium perfringens, and Brucella suis, as

247 well as the parasites Toxoplasma gondii, Sarcoystis cruzi, and Sarcoystis tenella. These

248 contaminants lead to a range of disorders, including polyradiculoneuritis in the case of

249 Campylobacter spp. infection. ${ }^{22-25}$ There have also been concerning reports of antimicrobial-

250 resistant pathogens found in commercial RMBDs, from strains of E. coli to various Salmonella

251 serovars, and there are ongoing reports of cats in the UK contracting Tuberculosis from a

252 particular brand of commercial RMBD. ${ }^{26-28}$ Many of these risks have been further explored by 
253 Davies et al in their 2019 review. ${ }^{2}$ One recent study found that only 63 out of 16,475 raw-feeding 254 households self-reported that a member of their household became ill due to raw products fed to 255 their pet(s), and of those households, only 39 had the pathogen confirmed by a laboratory. ${ }^{29}$ This 256 seems to indicate a very minimal human risk from feeding RMBDs to pets, however the two

257

258

259

260

261

262

263

264

265

266

267

268

269

270

271

272

273

274

275

276

277

278

279

280

281

282

283

284

285

286

287

288

289

290

291

292 most commonly reported pathogens in that study, Campylobacter and Salmonella are both considered to be widely underdiagnosed and underreported. ${ }^{30,31}$ It is difficult to gauge the exact degree to which zoonotic transmission of pathogens occurs from the feeding of RMBDs but the risk is certainly present. While responses from cooked feeders imply some level of understanding of the pathogenic risks of RMBDs, they also demonstrate a perception that commercial RMBDs are less risky than homemade RMBDs when there is little evidence to support this. By contrast, raw feeders were not likely to rate any type of RMBD as highly risky to either human or animal health. This correlates with Morelli et al who found that $65 \%$ of raw feeders believed that RMBD consumption cannot make dogs ill. ${ }^{15}$ These findings clearly indicate a need for further owner education on the pathogenic risks of RMDBs.

Interestingly, a quarter of cooked feeders $(n=45,25.1 \%)$ and roughly two-thirds of raw feeders $(n=13,65.3 \%)$ rated CCDs as highly risky when it comes to canine health. It is worth noting that participants were not given an opportunity to explain the reasoning behind their ratings, so it is unclear what perceived risks were being associated with CCDs. Cooked pet food can be host to microbial pathogens, however, the risk is significantly lower than for RMBDs; a study of over 1,000 commercially available pet foods found evidence of Salmonella or Listeria species in $40.8 \%$ of raw samples and only $0.42 \%$ of cooked ones. ${ }^{32}$ However there have been some highly publicized incidents of dry food recalls including a problem in 2007 with melamine contamination and more recently excess levels of vitamin D which may have been a factor for owners in answering this question. ${ }^{33,34}$ Exploring owner-perceived risks associated with commercially cooked food would be an interesting area for further research.

When respondents were asked to rate their veterinarian's knowledge of nutrition, $59.9 \%(\mathrm{n}=249)$ of owners surveyed believed their veterinarian to be highly knowledgeable (4 or 5 out of 5). A prior survey by Morgan et al found that only 35.9\% of surveyed dog owners trusted their veterinarians as a knowledge resource with respect to pet nutrition. ${ }^{5}$ While that statistic makes our figure seem surprisingly high, further analysis of our data shows a large difference in the assessment of veterinarian nutritional knowledge depending on the diet fed by the owner in question; cooked feeders gave their veterinarian an average score of 3.9 out of 5 while raw feeders gave an average score of only 2.9 out of 5 . The stark difference between the perceptions of the two groups demonstrates a need for better communication between veterinarians and their raw feeding clients, with specific regard to veterinarians' ability to inspire confidence in their own nutritional knowledge. Furthermore, this emphasizes the need for a strong foundation in nutrition in veterinary education alongside the communication skills and confidence to raise questions and start evidence-based discussions about nutrition in the consulting room. 
294 One of the novel aspects of this research was allowing owners to provide their opinions on raw

295

296

297

298

299

300

301

302

303

304

305

306

307

308

309

310

311

312

313

314

315

316

317

318

319

320

321

322

323

324

325

326

327

328

329

330

331

332

feeding in their own words using free-text boxes. When all owners were asked about the benefits of raw feeding, specific keywords repeatedly arose such as "teeth", "coat", "natural", "digestion" and "allergen", along with general positive words like "better" and "health". Highlighting the areas where owners think raw feeding benefits their dogs can serve as a starting point for veterinarians to open discussion about nutrition and help them to understand what owners find appealing about the practice of feeding RMBDs.

When asked why raw feeders chose to feed RMBDs, frequently repeated words were more often vague: "health", "better", "issues" and "benefits". These did not further the understanding of the motivation behind raw feeding and may expose an inability of raw feeders to agree on reasons to choose RMBDs. The lack of specific terms also emphasizes the dearth of peer-reviewed published data on the benefits of raw feeding.

Asking owners about the risks associated with raw feeding generated less ambiguous results, with words like "bacteria", "Salmonella", "contamination", "bone" and "unbalanced" being frequently repeated. This suggests that at least a subset of the population is informed about some of the documented risks of RMBDs. When owners who did not feed RMBDs were asked why they avoid the practice, they used tangible words again, with "time", "expensive", "risk", and "convenience" being some of the most repeated words. It is worth pointing out that the most common answers for why owners chose not to feed RMBDs do not directly correlate with the risks they listed previously. This perhaps indicates that it is not the risks associated with RMBDs that is preventing these owners from choosing that diet for their dog.

Compiling the free-text data into word clouds or tag clouds provides an intuitive and accessible way to visualize the data, rather than simply relying on frequency graphs. It demonstrates the complexity and diversity of data while highlighting the most important and frequently used keywords as the largest and centrally located words. ${ }^{35}$ The use of word clouds originated in the world wide web as a way to compile tags but has since evolved as a useful tool for text analytics. ${ }^{36}$ Their use in scientific literature has not been widespread, thus this application represents an innovative way to present our data. Our word clouds summarize a massive amount of free-text data and allow us to pick out trends and keywords quickly and efficiently while providing an aesthetically pleasing medium that is easy for a layperson to interpret.

The free-text boxes were a particular strength of this survey as it allowed owners a chance to express their perceptions in their own words. Additional strengths included our wide geographic spread of participants and variety in professional background of participants. Limitations of the survey include aspects that potentially restricted participation, in particular the need for respondents to be proficient in the English language as well as have internet and social media 
333

334

335

336

337

338

339

340

341

342

343

344

345

346

347

348

349

350

351

352

353

354

355

356

357

358

359

360

361

362

363

364

365

366

367

368

369

370

371

372

373

374

access. The varying geographical and cultural backgrounds of respondents may also have led to some confusion in terminology, for example, the term 'traditional' was used multiple times in the survey and yet will imply different connotations to a respondent depending on their background. As with many studies, sample size and participant engagement could have been more robust and will be a point of attention for future research.

\section{Conclusions}

This study used a novel questionnaire to assess dog owner perceptions around canine feeding of raw meat-based and commercial cooked diets. Dog owners have immense choice when deciding on a diet for their pets and the data indicated that an increasing number of them are choosing RMBDs, despite concerns cited by leading veterinary bodies. ${ }^{2}$ This contradiction indicates a clear need to understand what specifically is driving owners to choose RMBDs or CCDs, though it is equally important to assess what causes owners to avoid feeding these diet types too.

We found our hypotheses to be largely correct; dog owners who choose to feed RMBDs generally viewed the practice to be less risky to both human and dog health than owners who did not feed RMBDs. Raw feeders also rated raw diets as significantly more nutritious than CCDs. Moreover, over six in seven raw feeders perceived themselves as highly knowledgeable about nutrition, while only half viewed their veterinarian as knowledgeable. Conversely, cooked feeders perceived their veterinarians as more knowledgeable than they were about their dog's nutrition, but only half of them viewed CCDs as a nutritious diet. Further potential areas of research could include probing into specific claims made by raw feeders in the free-text portion of the questionnaire as well as exploring why cooked feeders choose their diet.

\section{Acknowledgments}

We would like to acknowledge Tim Parkin and William Houston for their help with statistics and coding.

\section{References}

1. Google Trend Data: "raw dog food." https://trends.google.com/trends/explore?date=200901-09 2019-01-09\&q=raw dog food.

2. Davies RH, Lawes JR, Wales AD. Raw diets for dogs and cats: a review, with particular reference to microbiological hazards. J Small Anim Pract. 2019;60(6):329-339. doi:10.1111/jsap. 13000

3. AVMA. Raw or Undercooked Animal-Source Protein in Cat and Dog Diets. 2019. https:/www.avma.org/policies/raw-or-undercooked-animal-source-protein-cat-and-dogdiets.

4. WSAVA. WSAVA Global Nutrition Committee Statement on Risks of Raw Meat-Based Diets. 2017. https://wsava.org/wp-content/uploads/2020/01/WSAVA-Global-NutritionCommittee-Statement-on-Risks-of-Raw-Meat.pdf.

5. Morgan SK, Willis S, Shepherd ML. Survey of owner motivations and veterinary input of owners feeding diets containing raw animal products. PeerJ. 2017. doi:10.7717/peerj.3031 
375 6. Freeman LM, Michel KE. Evaluation of raw food diets for dogs. J Am Vet Med Assoc.

376 2001;218(5):705-709.

377 7. Stockman J, Fascetti AJ, Kass PH, Larsen JA. Evaluation of recipes of home-prepared

378

379

380

381

382

383

384

385

386

387

388

389

390

391

392

393

394

395

396

397

398

399

400

401

402

403

404

405

406

407

408

409

410

411

412

413

414

415

416

417

418

419

420

8. Pedrinelli V, Gomes MDOS, Carciofi AC. Analysis of recipes of home-prepared diets for dogs and cats published in Portuguese. J Nutr Sci. 2017:1-5. doi:10.1017/jns.2017.31

9. Dillitzer N, Becker N, Kienzle E. Intake of minerals, trace elements and vitamins in bone and raw food rations in adult dogs. Br J Nutr. 2011;106(S1):S53-S56.

doi:10.1017/s0007114511002765

10. Taylor MB, Geiger DA, Saker KE, Larson MM. Diffuse osteopenia and myelopathy in a puppy fed a diet composed of an organic premix and raw ground beef. $J$ Am Vet Med Assoc. 2009. doi:10.2460/javma.234.8.1041

11. Krook L, Whalen JP. Nutritional secondary hyperparathyroidism in the animal kingdom: Report of two cases. Clin Imaging. 2010. doi:10.1016/j.clinimag.2010.08.010

12. Zeugswetter KF, Vogelsinger K, Handl S. Hyperthyroidism in dogs caused by consumption of thyroid-containing head meat. Schweiz Arch Tierheilkd. 2013;155(2):149152. doi:10.1024/0036-7281/a000432

13. Köhler B, Stengel C, Neiger-Casas R. Dietary hyperthyroidism in dogs. J Small Anim Pract. 2012. doi:10.1111/j.1748-5827.2011.01189.x

14. Dodd S, Cave N, Abood S, Shoveller AK, Adolphe J, Verbrugghe A. An observational study of pet feeding practices and how these have changed between 2008 and 2018. Vet Rec. 2020. doi:10.1136/vr. 105828

15. Morelli G, Bastianello S, Catellani P, Ricci R. Raw meat-based diets for dogs: Survey of owners' motivations, attitudes and practices. BMC Vet Res. 2019;15(1):1-10. doi:10.1186/s12917-019-1824-x

16. WSAVA Global Nutrition Committee. Recommendations on Selecting Pet Foods. https://wsava.org/wp-content/uploads/2020/01/Selecting-the-Best-Food-for-your-Pet.pdf. Published 2013.

17. Gruenberg W. Overview of Salmonellosis. In: MSD Veterinary Manuel. ; 2019. https://www.msdvetmanual.com/digestive-system/salmonellosis/overview-ofsalmonellosis.

18. Reimschuessel R, Grabenstein M, Guag J, Nemser SM, Song K, Qiu J, Clothier KA, Byrne BA, Marks SL, Cadmus K, Pabilonia K, Sanchez S, Rajeev S, Ensley S, Frana T, Jergens A, Chappell KH, Thakur K, Byrum B, Cui J, Zhang Y, Erdman MM, Rankin S, Daly R, Das S, Ruesch L, Lawhon SD, Zhang S, Baszler T, Diaz-Campos D, Hartmann F, Okwumabua O. Multilaboratory survey to evaluate Salmonella prevalence in diarrheic and nondiarrheic dogs and cats in the United States between 2012 and 2014. J Clin Microbiol. 2017. doi:10.1128/JCM.02137-16

19. Lefebvre SL, Reid-Smith R, Boerlin P, Weese JS. Evaluation of the risks of shedding Salmonellae and other potential pathogens by therapy dogs fed raw diets in Ontario and Alberta. Zoonoses Public Health. 2008;55(8-10):470-480. doi:10.1111/j.18632378.2008.01145.x

20. Lenz J, Joffe D, Kauffman M, Zhang Y, Lejeune J. Perceptions, practices, and consequences associated with foodborne pathogens and the feeding of raw meat to dogs. Can Vet J. 2009;50(6):637-643. 
421 21. Weese JS, Rousseau J. Survival of Salmonella Copenhagen in food bowls following

422

423

424

425

426

427

428

429

430

431

432

433

434

435

436

437

438

439

440

441

442

443

444

445

446

447

448

449

450

451

452

453

454

455

456

457

458

459

460

461

462

463

464

465

466 contanimation with experimentally inoculated raw meat: Effects of time, cleaning, and disinfection. Can Vet J. 2006.

22. Hellgren J, Hästö LS, Wikstrom C, Fernström LL, Hansson I. Occurrence of Salmonella, Campylobacter, Clostridium and Enterobacteriaceae in raw meat-based diets for dogs. Vet Rec. 2019. doi:10.1136/vr.105199

23. van Bree FPJ, Bokken GCAM, Mineur R, Franssen F, Opsteegh M, van der Giessen JWB, Lipman LJA, Overgaauw PAM. Zoonotic bacteria and parasites found in raw meat-based diets for cats and dogs. Vet Rec. 2018. doi:10.1136/vr.104535

24. van Dijk MAM, Engelsma MY, Visser VXN, Spierenburg MAH, Holtslag ME, Willemsen PTJ, Wagenaar JA, Borens EM, Roesty HIJ. . Brucella suis infection in dog fed raw meat, the Netherlands. Emerg Infect Dis. 2018. doi:10.3201/eid2406.171887

25. Martinez-Anton L, Marenda M, Firestone SM, Bushell RN, Child G, Hamilton AI, Long SI, Le Chevoir MAR. Investigation of the Role of Campylobacter Infection in Suspected Acute Polyradiculoneuritis in Dogs. J Vet Intern Med. 2018. doi:10.1111/jvim.15030

26. Finley R, Ribble C, Aramini J, Vandermeer M, Popa M, Litman M, Reid-Smith R. The risk of salmonellae shedding by dogs fed Salmonella-contaminated commerical raw food diets. Can Vet J. 2007.

27. Nilsson O. Hygiene quality and presence of ESBL-producing Escherichia coli in raw food diets for dogs . Infect Ecol Epidemiol. 2015. doi:10.3402/iee.v5.28758

28. O'Halloran C, Ioannidi O, Reed N, Murtagh, K, Dettermering E, Van Poucke S, Gale J, Vickers J, Burr P, Gascoyne-Binzi D, Howe R, Dobromylskyj M, Mitchell J, Hope J, Gunn-Moore D. Tuberculosis due to Mycobacterium bovis in pet cats associated with feeding a commercial raw food diet. J Feline Med Surg. 2019.

doi:10.1177/1098612X19848455

29. Anturaniemi J, Barrouin-Melo SM, Zaldivar-López S, Sinkko H, Hielm-Björkman A. Owners' perception of acquiring infections through raw pet food: A comprehensive internet-based survey. Vet Rec. 2019. doi:10.1136/vr.105122

30. Wagenaar JA, French NP, Havelaar AH. Preventing campylobacter at the source: Why is it so difficult? Clin Infect Dis. 2013. doi:10.1093/cid/cit555

31. WHO. WHO | Salmonella (non-typhoidal). World Heal Organ. 2015. https:/www.who.int/news-room/fact-sheets/detail/salmonella-(non-typhoidal).

32. Nemser SM, Doran T, Grabenstein M, McConnell T, McGrath T, Pamboukian R, Smith AC, Achen M, Danzeisen G, Kim S, Liu Y, Robseon S, Rosario G, McWilliams Wilson $\mathrm{K}$, Reimschuessel R. Investigation of listeria, salmonella, and toxigenic escherichia coli in various pet foods. Foodborne Pathog Dis. 2014. doi:10.1089/fpd.2014.1748

33. FDA. Melamine Pet Food Recall of 2007. 2018. https://www.fda.gov/animalveterinary/recalls-withdrawals/melamine-pet-food-recall-2007.

34. FDA. Vitamin D Toxicity in Dogs. https://www.fda.gov/animal-veterinary/animal-healthliteracy/vitamin-d-toxicity-dogs.

35. Bateman S, Gutwin C, Nacenta MA. Seeing Things in the Clouds: The Effect of Visual Features on Tag Cloud Selections. Proc ACM Conf Hypertext Hypermedia (Hypertext '08). 2008:193-202. doi:10.1145/1379092.1379130.

36. Heimerl F, Lohmann S, Lange S, Ertl T. Word cloud explorer: Text analytics based on word clouds. In: Proceedings of the Annual Hawaii International Conference on System Sciences. IEEE; 2014:1833-1842. doi:10.1109/HICSS.2014.231 
Figure 1

Owner responses to rating questions

Dog owner responses when asked to rate cooked diets or raw diets on three variables

(nutritional value, risk to dog health, or risk to human health) as well as their own knowledge of nutrition and their veterinarian's knowledge of nutrition on a 5-point scale, where 1 was the least and 5 was the most of each variable, separated by owner's chosen diet (cooked feeders versus raw feeders).

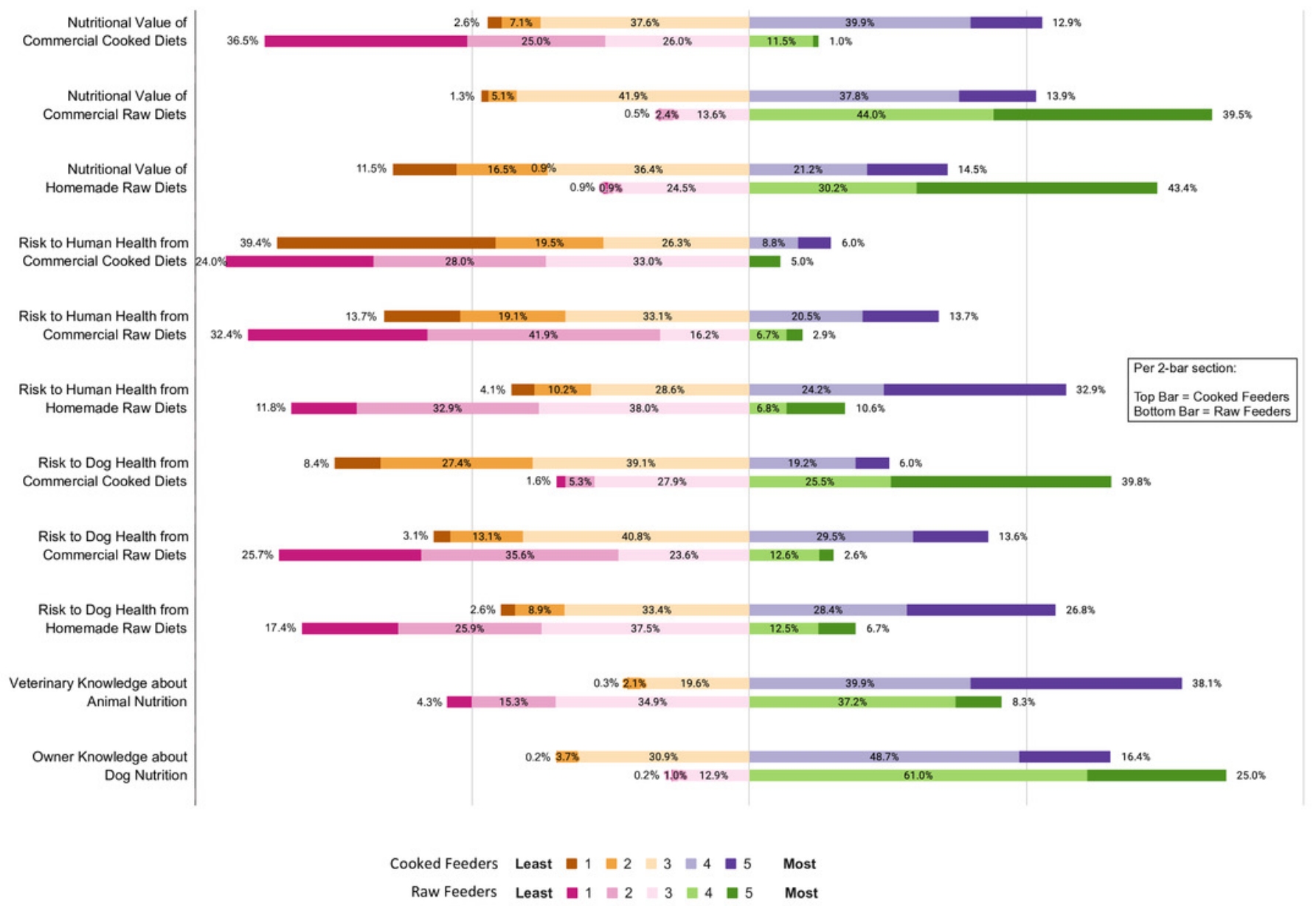


Figure 2

Perceived benefits of raw diets

Response to the prompt: What benefits are you aware of associated with feeding a raw diet?

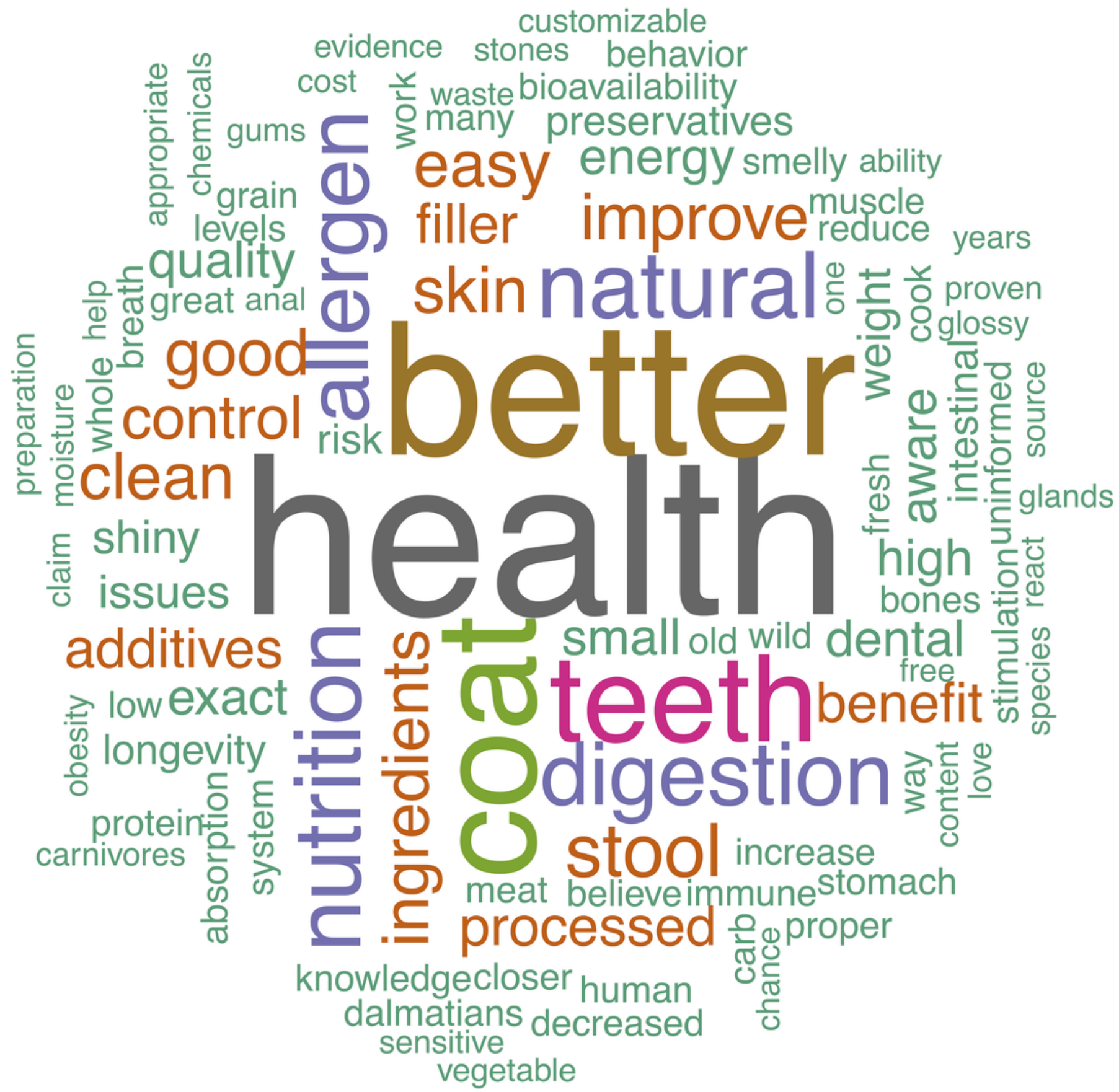


Figure 3

Perceived risks of raw diets

Response to the prompt: What risks are you aware of associated with feeding a raw diet?

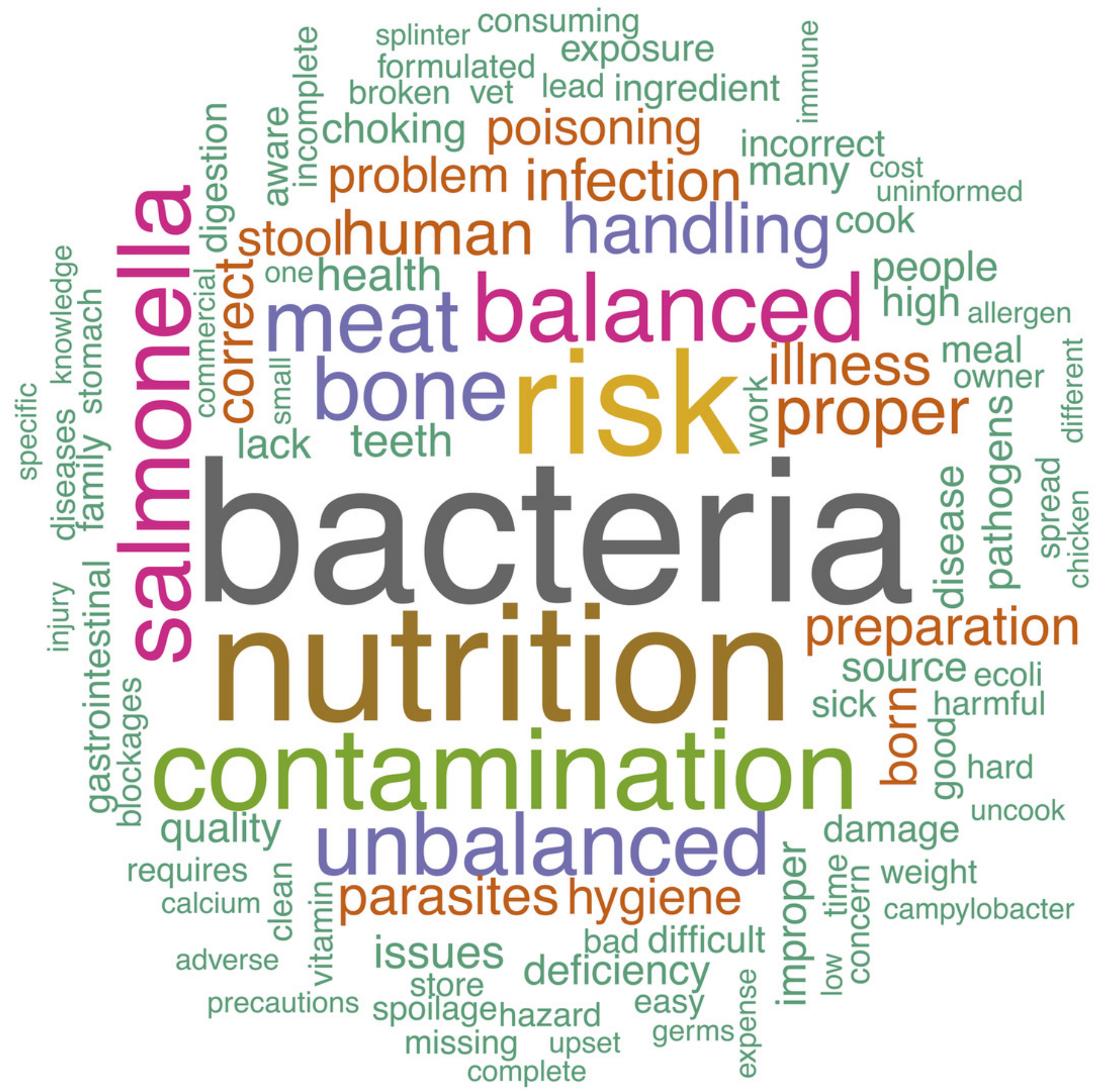


Figure 4

Reasons for feeding a raw diet.

Response to the prompt: If you feed your dog a raw diet, why?

system

dental preservatives

based joints balanced

way many processed condition naturalimprove quality low meal lab. . helpstooCommercial old s skin best controlmeatgreat fresh > provide

digestion

vet $\stackrel{\complement}{\oplus}$ bone $₫$

research

previous (1)

previous $\stackrel{1}{2}$

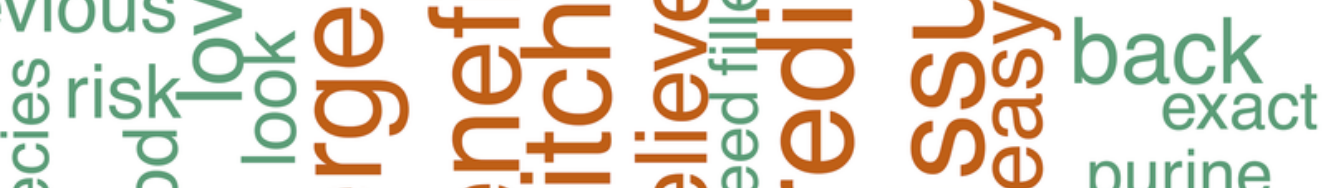

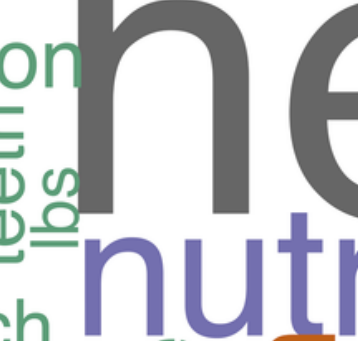

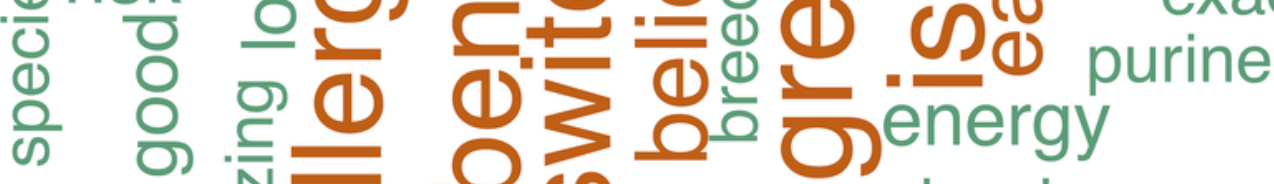

ฮิ gastrointestinal 
Figure 5

Reasons for not feeding a raw diet.

Response to the prompt: If you do not feed your dog a raw diet, why not?

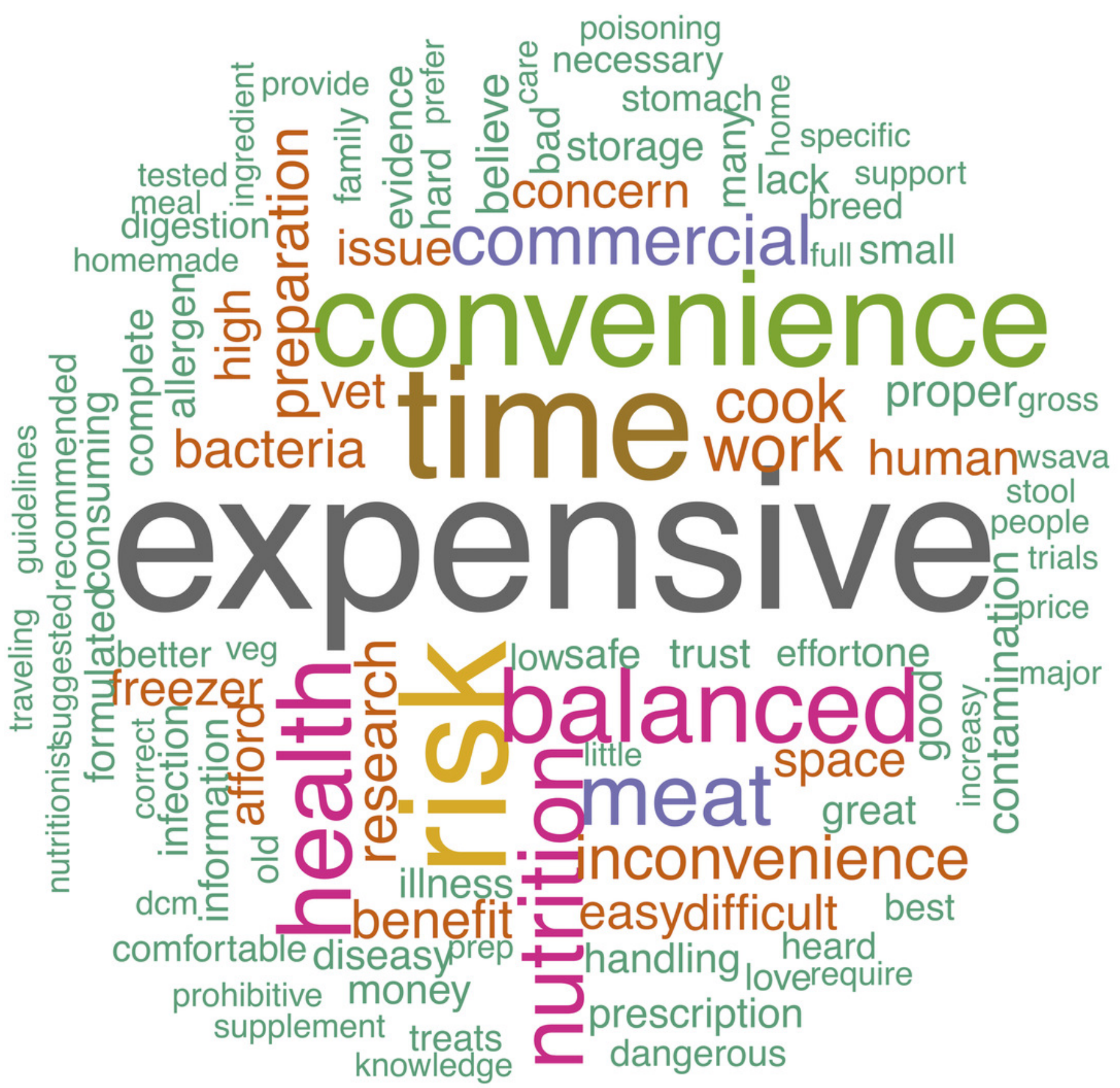


Table $\mathbf{1}$ (on next page)

Demographics.

Demographic data for survey respondents. 
1

\begin{tabular}{|c|c|c|}
\hline Variable & Number of respondents & $\%$ \\
\hline Total survey respondents & 419 & 100 \\
\hline \multicolumn{3}{|l|}{ Gender } \\
\hline Apogender & 1 & 0.2 \\
\hline Female & 393 & 93.6 \\
\hline Male & 23 & 5.5 \\
\hline Nonbinary & 1 & 0.2 \\
\hline Prefer not to say & 2 & 0.5 \\
\hline \multicolumn{3}{|l|}{ Age (years) } \\
\hline $18-24$ & 60 & 14.3 \\
\hline $25-35$ & 146 & 34.8 \\
\hline $36-45$ & 60 & 14.3 \\
\hline $46-55$ & 71 & 16.9 \\
\hline $56-65$ & 64 & 15.3 \\
\hline Over 65 & 18 & 4.3 \\
\hline \multicolumn{3}{|l|}{ Country of Residence } \\
\hline Australia & 11 & 3.1 \\
\hline Canada & 25 & 7.0 \\
\hline New Zealand & 2 & 0.6 \\
\hline Singapore & 2 & 0.6 \\
\hline South Africa & 1 & 0.3 \\
\hline Spain & 1 & 0.3 \\
\hline Turkey & 1 & 0.3 \\
\hline United Kingdom & 97 & 27.2 \\
\hline United States & 206 & 57.9 \\
\hline $\begin{array}{l}\text { Other: One respondent from each country (China, France, Hong } \\
\text { Kong, Italy, Malaysia, Norway, Portugal, South Africa, Spain, } \\
\text { Turkey) }\end{array}$ & 10 & 2.8 \\
\hline \multicolumn{3}{|l|}{ Industry (Animal-related field?) } \\
\hline Animal Industry (pet food, toys, products) & 12 & 2.9 \\
\hline $\begin{array}{l}\text { Animal Services (groomer, farrier, acupuncturist, kennel staff, } \\
\text { etc.) }\end{array}$ & 37 & 8.9 \\
\hline Breeder & 5 & 1.2 \\
\hline No. Other profession not related to animal industry & 306 & 73.2 \\
\hline Student of veterinary medicine/science/nursing & 34 & 8.1 \\
\hline Veterinarian or Vet Nurse & 24 & 5.7 \\
\hline \multicolumn{3}{|l|}{ Owner's Dietary Preferences } \\
\hline Omnivore (meat and plant-based diet) & 357 & 85.6 \\
\hline Vegan & 9 & 2.2 \\
\hline Vegetarian & 39 & 9.4 \\
\hline Other & 12 & 2.9 \\
\hline \multicolumn{3}{|l|}{ Household with children under 10 years of age } \\
\hline No & 375 & 89.7 \\
\hline Yes & 43 & 10.3 \\
\hline \multicolumn{3}{|l|}{ Household with immunocompromised individual } \\
\hline "I don't know" & 8 & 1.9 \\
\hline No & 376 & 90.2 \\
\hline Yes & 33 & 7.9 \\
\hline \multicolumn{3}{|l|}{ Household with pregnant individual } \\
\hline "I don't know" & 1 & 0.2 \\
\hline No & 408 & 97.4 \\
\hline Yes & 10 & 2.4 \\
\hline
\end{tabular}

